Волокитина И. Е. Панин Е. А.

Волокитин А. В.

\title{
ИЗУЧЕНИЕ ДЕФОРМИРОВАННОГО СОСТОЯНИЯ ПРИ ПЛАСТИЧЕСКОЙ ОБРАБОТКЕ МЕТОДОМ КРУЧЕНИЯ ПОД ВЫСОКИМ ДАВЛЕНИЕМ
}

Проектирование технологических процессов обработки металлов давлением на современном уровне предполагает решение целого ряда весьма сложных задач. Основная цель состоит в том, чтобы выявить закономерности процесса, существенные факторы и параметры, влияющие на его протекание, оценить их возможные воздействия на эксплуатационные свойства получаемых изделий [1-3]. Полученные данные позволяют сформировать информационное поле конкретной технологии и, таким образом, иметь возможность управления, как процессом, так и свойствами изделий.

Компьютерное моделирование позволяет получить большой объем информации, провести всестороннее исследование процесса пластического деформирования, определить его особенности, рассмотреть и сопоставить большое количество альтернативных вариантов технологических процессов. Весьма большими возможностями в этом плане обладает компьютерная программа DEFORM, которая предназначена для моделирования сложных трехмерных процессов пластического деформирования металлов, таких как ковка, объемная штамповка, волочение, прокатка, прессование и относится к технологическим САЕ-системам (системам инженерных расчетов). Программа позволяет проследить за течением металла и получить макроструктуру металлоизделия, минимизировать усилие деформирования, подобрать оборудование, оптимизировать количество технологических переходов, моделировать образование дефектов и помочь понять способы их устранения, прогнозировать механические свойства конечного изделия, микроструктуру и фазовый состав, рассчитать на прочность деформирующий инструмент. Кроме того, при помощи моделирования можно определить также важные параметры заготовки, как накопленная деформация, напряжение, температура, направление волокна заготовки, возможность разрушения заготовки, износ инструмента [4].

DEFORM - специализированный инженерный программный комплекс, предназначенный для анализа процессов обработки металлов давлением, термической и механической обработки. DEFORM позволяет проверить, отработать и оптимизировать технологические процессы непосредственно за компьютером, а не в ходе экспериментов на производстве методом проб и ошибок. Благодаря этому существенно сокращаются сроки выпуска продукции, повышается ее качество и снижается себестоимость [5].

Алгоритм, используемый для расчета в конечно-элементном пакете DEFORM, предполагает выполнение следующих расчетных процедур:

1. Создание геометрии заготовки и деформирующего инструмента. Существуют два основных способа задания геометрии инструмента и заготовки: использование так называемых булевых операций непосредственно в программном комплексе DEFORM 3D и создание двух/трехмерных моделей в специальных автоматизированных программах для инженерного проектирования. Использование специальных автоматизированных программ позволяет строить более сложные модели, так как они оснащены специальными инструментами, обеспечивающими создание практически любых конфигураций модели. Для создания моделей (геометрии) заготовки и оснастки использовалась система автоматизированного проектирования КОМПАС-3D. Создание моделей осуществлялось с учетом геометрического подобия. 
2. Создание сетки конечных элементов и установка свойств заготовки и инструмента. Величина конечных элементов влияет на точность расчета. Деформируемое тело в модели было определено сеткой тетрагональных конечных элементов, распределенных в объеме заготовки. Общее количество конечных элементов заготовки выбрано равным 60 000, что позволяет производить расчет с достаточной точностью при размере заготовки диаметром 30 мм и толщиной 10 мм. После построения сетки задается материал заготовки и инструмента.

Механическое поведение деформируемого тела описано моделью пластического поведения, инструмент является жестким (недеформируемым). Материал оснастки и заготовки задавался выбором из базы данных программы. Для заготовок выбран - титан. Моделирование осуществлялось с учетом теплообмена (теплопередачи) между заготовкой, оснасткой и окружающей средой при нормальных условиях (температура окружающей среды принята равной $20^{\circ} \mathrm{C}$ ). Для инструмента выбрана комнатная температура.

3. Выбор граничных условий. Условия трения на поверхности контакта во многом определяют процесс протекания деформирования и соответственно силовые параметры и напряженно-деформированное состояние в очаге деформации. В данной работе коэффициент трения выбран равным 0,3 на основании ранее проведенных исследований [6-8].

4. Назначение движения. При установке движения определяется главный инструмент, который будет осуществлять деформирование (в нашем случае верхний боек), и задается для него направление вращения и скорость движения. Для верхнего бойка задана постоянная скорость движения вдоль оси z.

5. Установка условий расчета и расчет.

Для реализации процесса кручения под высоким давлением на существующем оборудовании лаборатории кафедры ОМД была разработана матрица специальной конструкции, позволяющая реализовать процесс скручивания под высоким давлением, за счет прямолинейного движения бойка относительно станины (рис. 1).

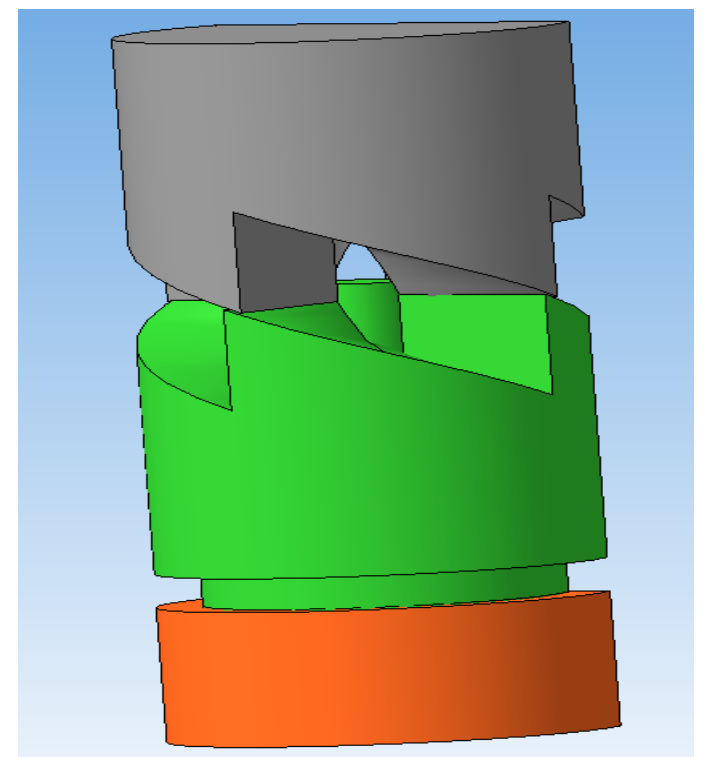

Рис. 1. Модель матрицы для кручения под высоким давлением

Прямолинейное движение верхнего бойка, с закрепленной на нем верхней частью матрицы, за счет сил контактного трения направленных под наклонным углом к ответной части матрицы, передает ей крутящий момент, в результате чего прямолинейное движение превращается в движение кручения.

Матрица состоит из трех частей. Нижней части, представляющей собой неподвижно закрепленную матрицу, в которую помещается образец в виде диска диаметром 30 мм и толщиной 10 мм. Вращающаяся часть матрицы, имеющая нижнюю плоскую поверхность, контактирующую с заготовкой. И верхнюю поверхность, представляющую собой 
спиралевидную форму, состоящей из четырех сегментов. Верхняя часть матрицы неподвижно закреплена в верхнем бойке, также имеющем спиралевидную форму, состоящем из четырех сегментов.

В начальный момент сегменты разъединены, далее происходит сближение верхней и нижней частей матрицы. За счет сегментов матрицы, расположенных под острым углом, и спиралевидной формы происходит закручивание центральной части матрицы и непосредственно давление на образец.

Температура заготовки, как и температура матрицы, была выбрана равной $20{ }^{\circ} \mathrm{C}$ - для получения оптимальных значений параметров НДС и усилий деформирования.

Для проверки реализации способа вышеназванный процесс был смоделирован в пакете Deform 2D и 3D, что позволило выявить «слабые места» процесса, оценить напряженнодеформированное состояние заготовки на каждом переходе цикла, интенсивность деформации, полученную за один полный цикл данного способа, а также потребное усилие деформирования. Все эти факторы необходимы для оценки реализации способа, а также проектирования технологической оснастки, выбора силового оборудования для проведения эксперимента, на основании которого можно судить о степени изменения структуры материала заготовки.

Напряженно-деформированное состояние (НДС) при деформировании было проанализировано по результатам распределения:

1) интенсивности логарифмической деформации (strain effective) $-\varepsilon_{\text {инт; }}$;

2) эквивалентного напряжения (stress effective) $-\sigma_{\text {экв; }}$

3) гидростатического давления или среднего напряжения (stress mean) $-\sigma_{\mathrm{cp}}$.

1. Интенсивность логарифмической деформации (strain effective).

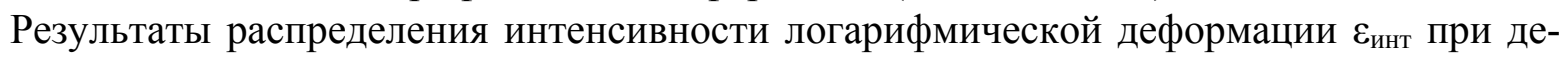
формировании методом кручения под высоким давлением представлено на рис. 2.

В результате моделирования определены интенсивности логарифмической деформа-

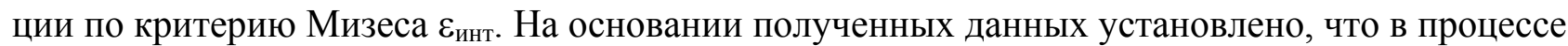
деформирования наиболее высокая логарифмическая деформация наблюдается по контурам заготовки, что объясняется влиянием сил внешнего трения. Анализируя НДС разрезов полученных образцов, было выявлено, что распределение деформации по всему объему деформируемой заготовки носит весьма равномерный характер. Причем степень деформирования внутренних слоев практически не отличается от степени деформирования поверхностных об этом можно судить по однородной окраске внутренних и внешних слоев на разрезе.

2) эквивалентные напряжения (stress effective)

Напряженное состояние заготовки в момент деформирования является важнейшей характеристикой, влияющей на получение качественного металла.

Результаты распределения эквивалентных напряжений бэкв при КВД представлены на рис. 3.

Эквивалентное напряжение охватывает весь очаг деформации и достигает величины в центральных слоях заготовки до 450 МПа и до 630 МПа во внешних слоях, поскольку материал во внешней области течет быстрее, чем материал центральной зоны (рис. 4).

3. Гидростатическое давление или среднее напряжение (stress mean) $-\sigma_{\mathrm{cp}}$.

Важным фактором, оказывающим существенное влияние на эффективность структурообразования, особенно для малопластичных и труднодеформируемых материалов, таких как титан, является величина гидростатического давления. Наложение гидростатического давления обеспечивает высокую однородность распределения напряжений, деформаций и структурного состояния, способствует созданию благоприятных условий контактного трения, сохранению ресурса пластичности [9]. 


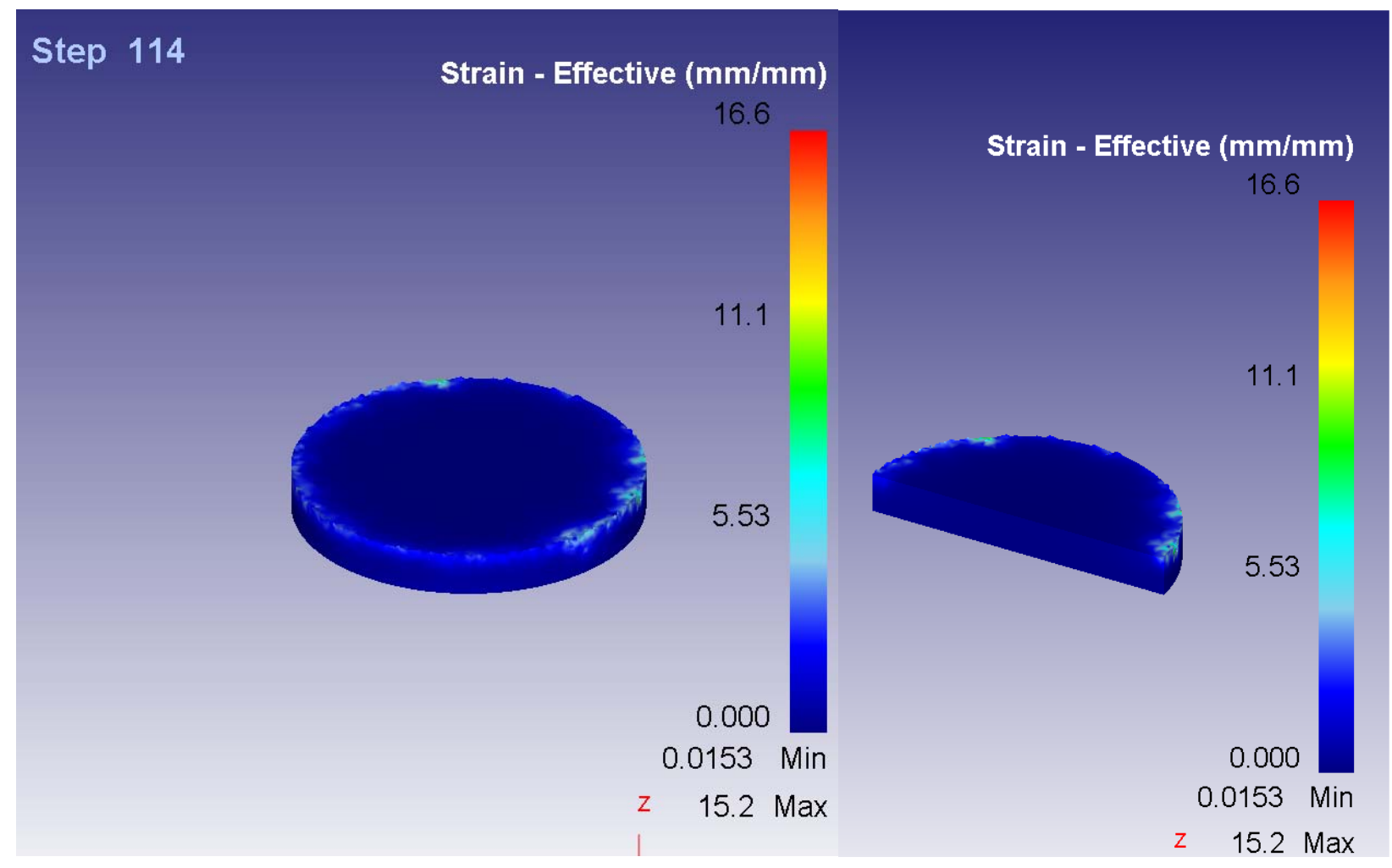
заготовки

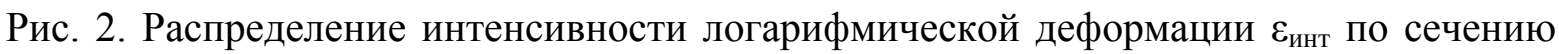

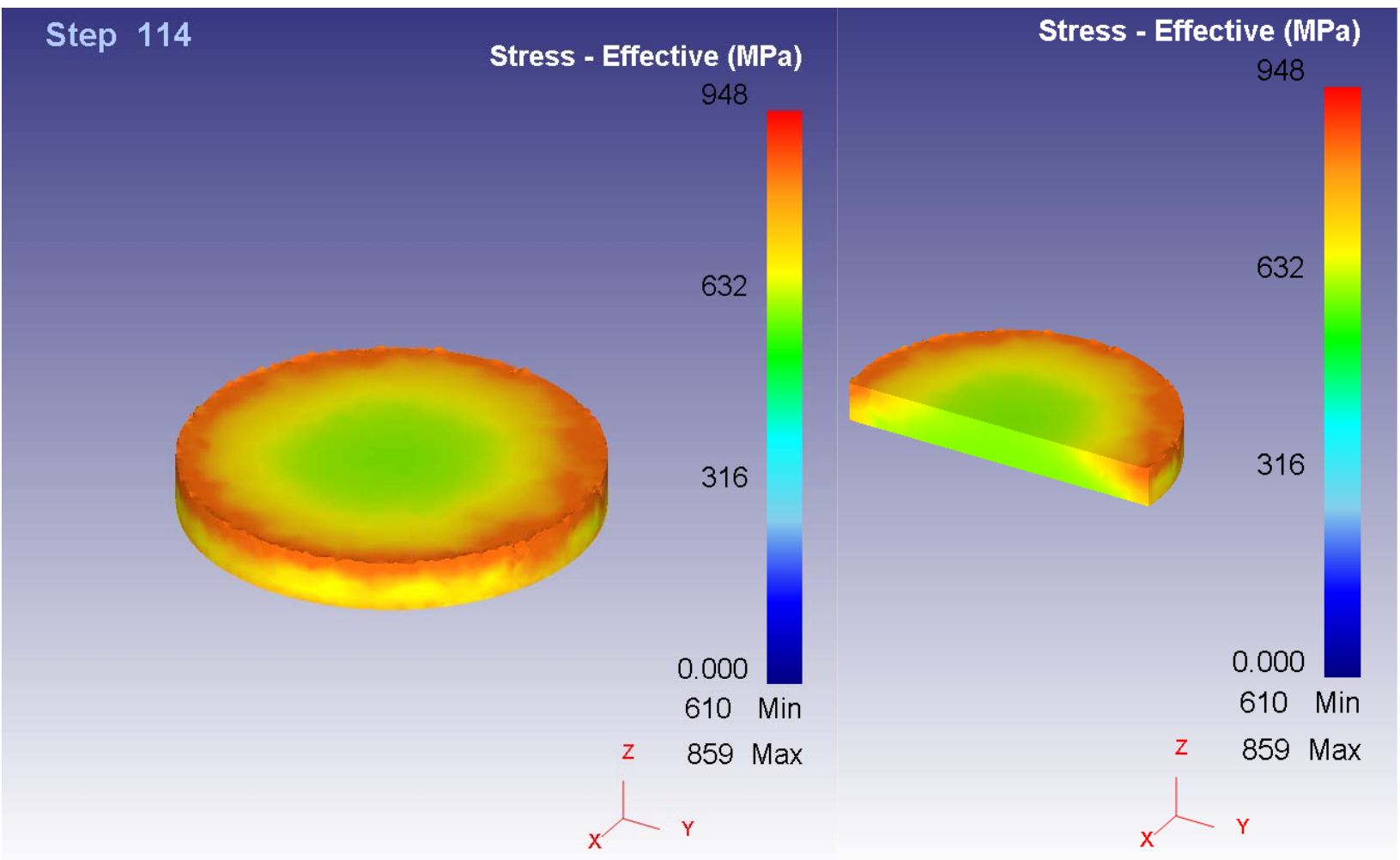

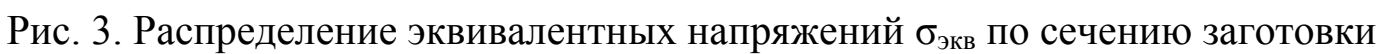




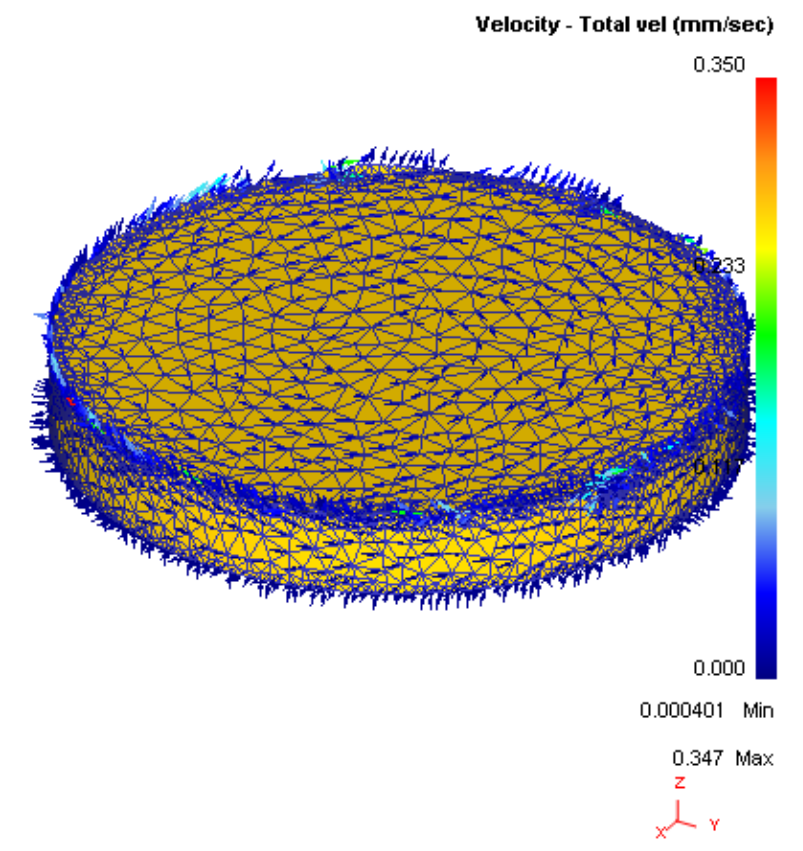

Рис. 4. Векторное поле скоростей

На качество металла, например, на лучшую проработку литой структуры и заваривание внутренних дефектов, положительно влияет наличие сжимающих напряжений внутри деформируемого тела, возникающих в процессе прессования. Эти напряжения можно оха-

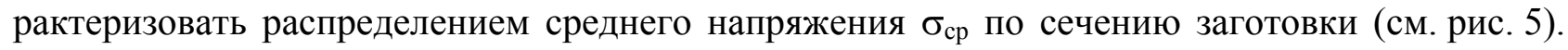
Чем больше абсолютная величина среднего давления сжатия (отрицательного гидростатического давления), тем выше пластичность металла и чем меньшую роль в схеме главных напряжений играют растягивающие напряжения, тем большую способность к пластической деформации проявляет металл. Высокое гидростатическое давление существенно увеличивает деформируемость материалов. Известно также, что высокое гидростатическое давление активизирует дислокационное скольжение, подавляет процессы возврата, приводит к фрагментации структуры при меньших, чем обычно, степенях деформации.

Характер распределения $\sigma_{c p}$ показывает, что в очаге деформации, за исключением небольших зон, преобладают сжимающие напряжения. Максимальные главные напряжения заготовки составляют не более $1080 \mathrm{MПа,} \mathrm{распределение} \mathrm{максимальных} \mathrm{главных} \mathrm{напряже-}$ ний сконцентрированы в основном на периферии заготовки.

После проведения анализа с использованием инструмента «Damage» было выявлено, что опасности разрушения заготовки нет даже после проведения десяти циклов деформирования.

Для получения ультрамелкозернистой структуры деформирование было решено проводить при комнатной температуре, но в процессе деформации за счет сил трения заготовка разогревается до температуры $135{ }^{\circ} \mathrm{C}$. Холодное кручение под высоким давлением сопровождается выделением большого количества тепла и повышением температуры материала в очаге деформации. В процессе деформирования тепло выделяется из-за внутреннего трения (пластической деформации, в результате которой происходит трение одних частей металла о другие). Около 85 \% механической работы, используемой при кручении, затрачивается на преодоление внутреннего трения, в тепло обращается и вся работа внешнего трения.

Для титана такой разогрев не приведет к возникновению рекристаллизации, а поможет частично снимать напряжения во время деформирования, за счет чего можно провести большее количество циклов кручения. Проведя анализ рис. 6, можно сказать, что разогрев заготовки происходит равномерно по всему сечению.

При разработке технологических процессов обработки металлов давлением и проектировании оборудования необходимо знать энергосиловые параметры процесса, в частности 
усилие, которое нужно приложить к деформируемому телу для преодоления сопротивления металла деформации и трения на поверхности контакта металла с инструментом. Следовательно, по результатам моделирования был построен график изменения усилия деформирования (см. рис. 7).

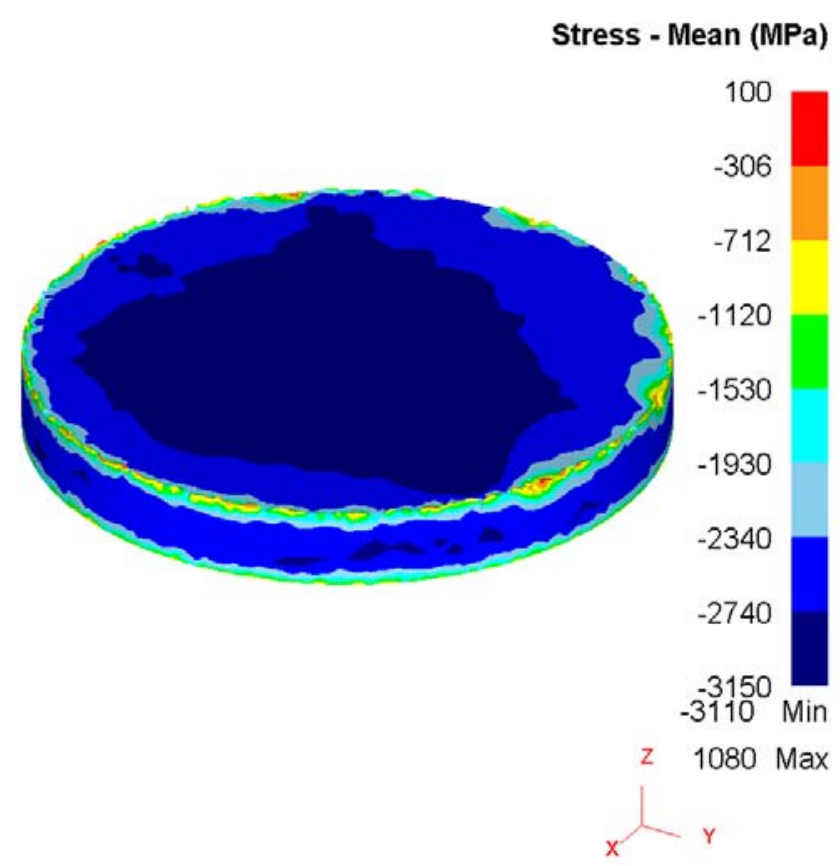

Рис. 5. Распределение среднего напряжения $\sigma_{\mathrm{cp}}$ по сечению заготовки

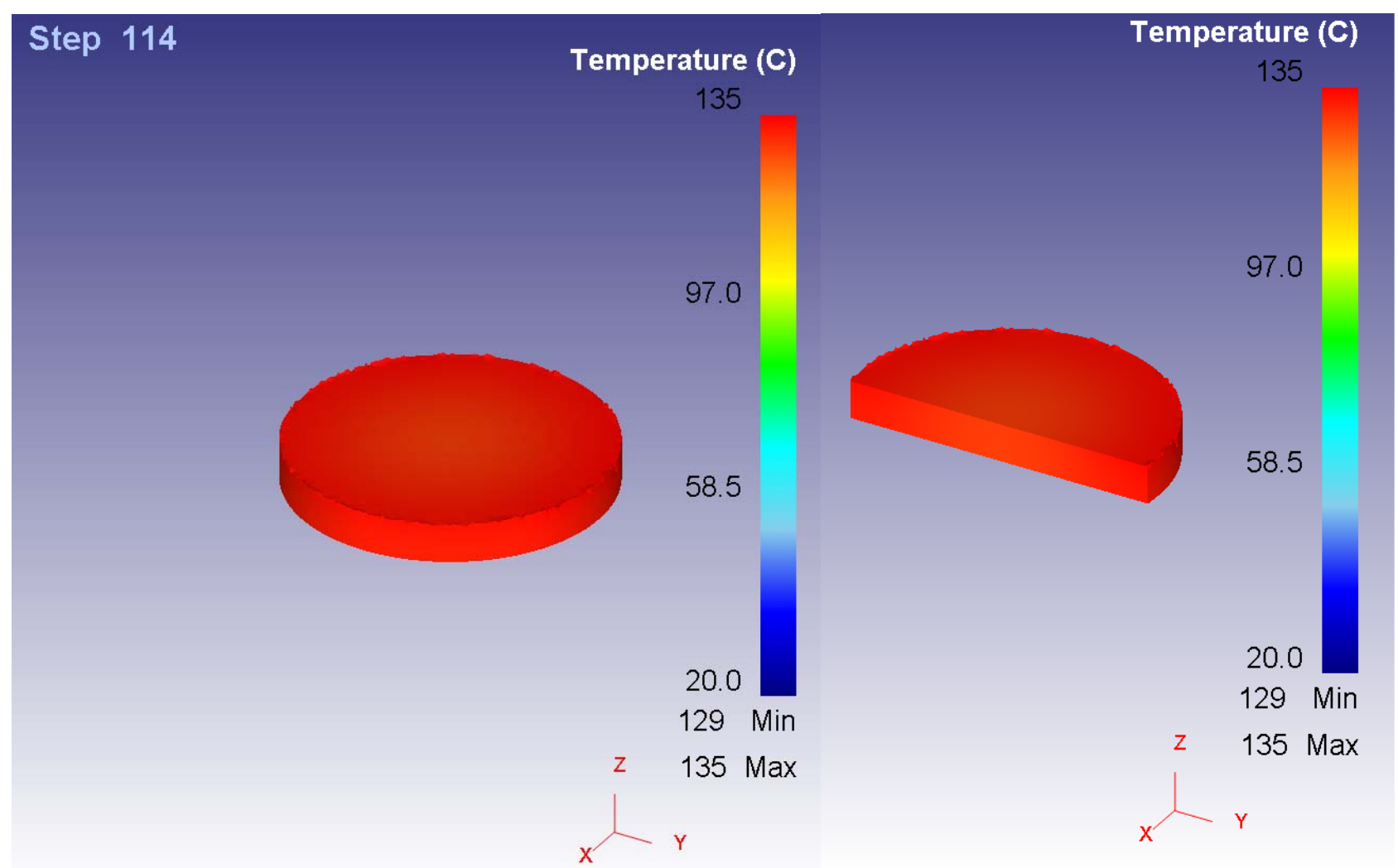

Рис. 6. Распределение температуры по сечению заготовки 


\section{Load Prediction}

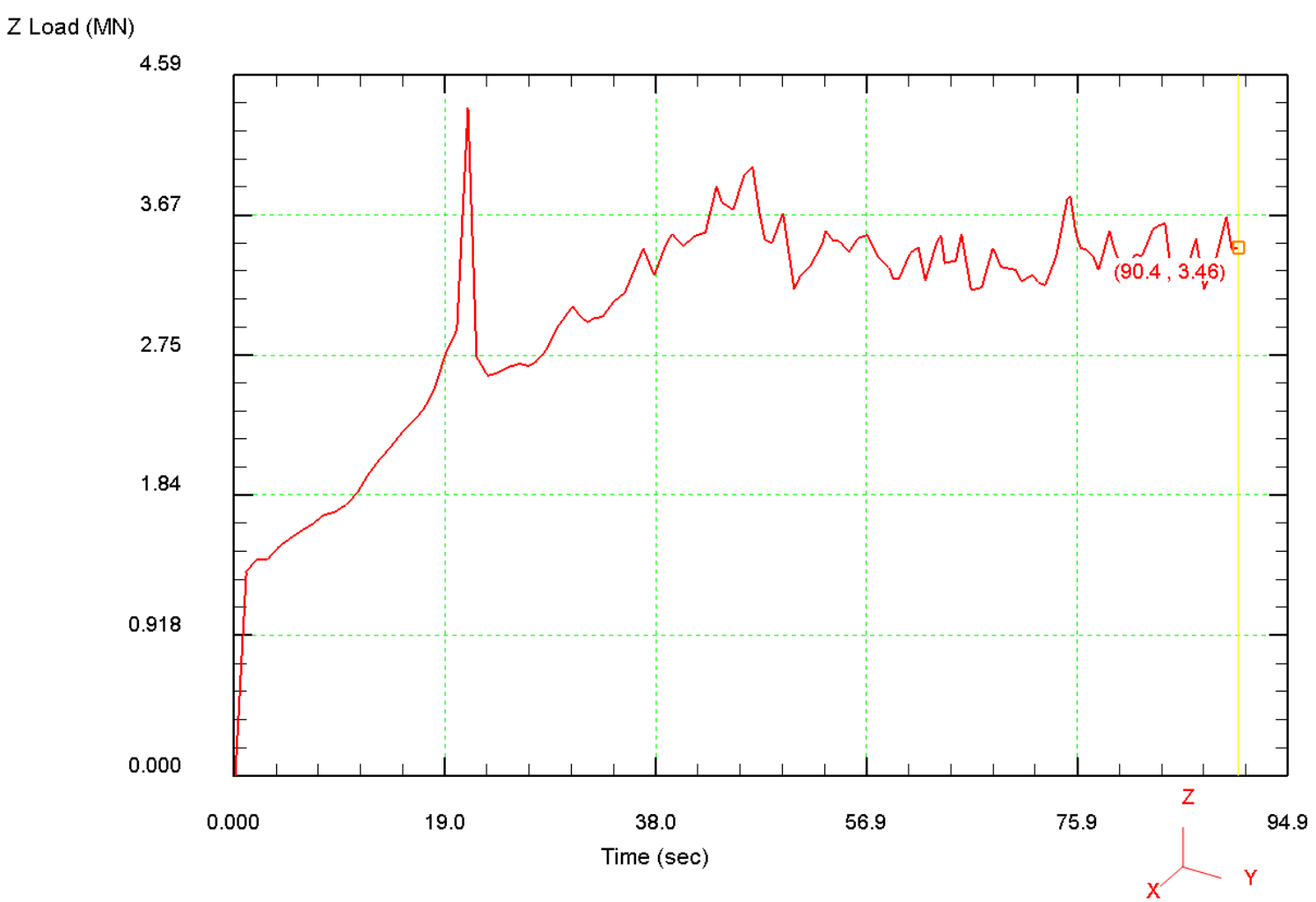

Рис. 7. Величина усилия деформирования при кручении под высоким давлением

После моделирования исследуемого процесса, было оценено усилие деформирования, которое составило порядка 3,5 MH (рис. 7), а также интенсивность деформаций и напряжений, рост которых приводит к измельчению микроструктуры деформируемого материала.

Анализируя полученные результаты, можно сделать вывод о том, что наблюдается равномерное увеличение усилия деформирования, что объясняется пластической деформацией образца, степень которой достигает $1 \%$. Максимальное пиковое напряжение соответствует максимальному упрочнению образца. Далее прослеживается скачкообразное изменения усилия деформирования связанное с процессами кручения заготовки под высоким давлением.

\section{ВЫВОДЫ}

В статье проведено моделирование процесса деформирования в программном комплексе Deform 3D, в результате которого можно сделать следующие выводы:

- на основании анализа НДС показана возможность обеспечения деформаций сдвига в обрабатываемом металле;

- в результате изучения модели с несколькими циклами деформирования было выявлено, что с увеличением числа проходов происходит не только общее снижение среднего диаметра зерна, но и постепенное выравнивание данного параметра между центральной и поверхностной зоной.

\section{СПИСОК ИСПОЛЬЗОВАННОЙ ЛИТЕРАТУРЫ}

1. Лежнев С.Н., Кривцова О. Н., Ибатов М. К., Самодурова М., Панин Е.А., Моделирование напряженно-деформированного состояния прокатных валков. Обработка материалов давлением. Краматорск: ДГМА. 2013. 3 (36). C. 86-89. 
2. Найзабеков А.Б., Лежнев С.Н., Панин Е.А., Крупенькин И.И. Оценка эффективности новой энергосберегающей технологии прокатки толстого листа на основе компьютерного моделирования. Обработка материалов давлением. Краматорск: ДГМА. 2016. 2 (43). С. 104-110.

3. Shaeri M.H., Salehi M.T., Seyyedein S.H., Abutalebi M.R., Park J.K. Microstructure and mechanical properties of Al-7075 alloy processed by equal channel angular pressing combined with aging treatment. Materials and Design. 2014, 57, pp. 250-257.

4. Каргин В.Р., Каргин Б.В., Ерисов Я.А. Моделирование прессования в программе DEFORM-2D. Caмара. 2010. $106 \mathrm{c}$.

5. Сотов А.В., Смелов В.Г., Агаповичев А.В., Карташов Р.Д. Моделирование процессов ОМД в программном комплексе Deform: методические указания. Самара: Изд-во Самар. ун-та. 2017. 47 с.

6. Ahmed Drai. Analysis of the temperature effect on the plastic strain of polymers during high pressure torsion (HPT) process. The Eurasia Proceedings of Science, Technology, Engineering \& Mathematics (EPSTEM). 2013, V. 1, pp. 41-51.

7. Aour B., Zaïri F., Gloaguen J.M., Naït-Abdelaziz M., Lefebvre J.M. A computational study of die geometry and processing conditions effects on equal channel angular extrusion of a polymer. International journal of mechanical sciences. 2008. 50, pp. 589-602.

8. Mousumi D., Goutam D., Mainak G., Matthias W., Rajnikant V., Ghosh C.S. Microstructures and mechanical properties of HPT processed 6063 Al alloy. Materials Science \& Engineering. 2012. A558, pp. 525-532.

9. Рыбин Ю.И., Рудской А.И., Золотов А.М. Математическое моделирование и проектирование технологических процессов обработки металлов давлением. СПб.: Наука. 2004. 644c.

\section{REFERENCES}

1. Lezhnev S.N., Krivtsova O.N., Ibatov M.K., Samodurova M., Panin E.A., Modeling of the stress-strain state of rolling rolls. Materials Working by Pressure. Kramatorsk: DSEA. 2013. 3 (36), pp. 86-89. (in Russian).

2. Naizabekov A.B., Lezhnev S.N., Panin E.A., Krupenkin I.I. Evaluation of the effectiveness of new energysaving technology for rolling a thick plate based on computer simulation. Materials Working by Pressure. Kramatorsk: DSEA. 2016. 2 (43), pp. 104-110. (in Russian).

3. Shaeri M.H., MSalehi.T., Seyyedein S.H., Abutalebi M.R., Park J.K. Microstructure and mechanical properties of Al-7075 alloy processed by equal channel angular pressing combined with aging treatment. Materials and Design. 2014, 57, pp. 250-257. (in Russian).

4. Kargin V.R., Kargin B.V., Erisov Y.A. Press modeling in the DEFORM-2D program. Samara. 2010, 106 p.

5. Sotov A.V., Smelov V.G., Agapovichev A.V., Kartashov R.D. Modeling metal forming processes in the Deform software package. Samara: Publishing house Samara University. 2017, 47 p. (in Russian).

6. Ahmed Drai. Analysis of the temperature effect on the plastic strain of polymers during high pressure torsion (HPT) process. The Eurasia Proceedings of Science, Technology, Engineering \& Mathematics (EPSTEM). 2013, 1, pp. 41-51.

7. Aour B., Zairi F., Gloaguen J.M., Nait-Abdelaziz M., Lefebvre J.M. A computational study of die geometry and processing conditions effects on equal channel angular extrusion of a polymer. International journal of mechanical sciences. 2008, 50, pp. 589-602.

8. Mousumi D., Goutam D., Mainak G., Matthias W., Rajnikant V., Ghosh C.S. Microstructures and mechanical properties of HPT processed 6063 Al alloy. Materials Science \& Engineering. 2012. A558, pp. 525-532.

9. Rybin Yu.I., Rudskoy A.I., Zolotov A.M. Mathematical modeling and design of technological processes of metal forming. St. Petersburg: Nauka. 2004, 644 p. (in Russian).

Волокитина И. Е. - $\mathrm{PhD}$, доцент. КГИУ;

Панин Е. А. $\quad-\mathrm{PhD}$, доцент. КГИУ;

Волокитин А. В. - $\mathrm{PhD}$, ст. преп. КГИУ.

КГИУ - Карагандинский государственный индустриальный университет, г. Темиртау, Казахстан.

E-mail: irinka.vav@mail.ru 